\title{
Forensische Modelle zur Gewaltprävention an der Schnittstelle zur Allgemeinpsychiatrie: der forensisch-psychiatrische Konsildienst
}

\author{
Catharina Schmidt ${ }^{1}$ Joachim Nitschke ${ }^{2}$ Elmar Habermeyer ${ }^{1}$ \\ Eingegangen: 11. Juni 2021 / Angenommen: 20. Juni 2021 / Online publiziert: 20. Juli 2021 \\ ○ Der/die Autor(en) 2021
}

\section{Zusammenfassung}

Obwohl bei einer Untergruppe psychisch kranker Menschen ein erhöhtes Gewaltrisiko verortet werden kann und Patienten der Maßregelvollzugskliniken in der Regel eine allgemeinpsychiatrische Vorgeschichte haben, existieren bislang nur vereinzelt präventiv ausgerichtete Behandlungsmodelle an der Schnittstelle zwischen forensischer und allgemeiner Psychiatrie. Im vorliegenden Artikel werden unterschiedliche Ansätze einer interdisziplinären Zusammenarbeit vorgestellt und das Angebot des forensisch-psychiatrischen Konsildiensts der Fachstelle Forensic Assessment \& Risk Management (FFA) der Klinik für Forensische Psychiatrie (KFP) der Psychiatrischen Universitätsklinik Zürich (PUK) beschrieben.

Schlüsselwörter Risikomanagement · Forensisches Fachwissen · Präventionsambulanz · Risikofaktoren · Unterstützung der Allgemeinpsychiatrie

\section{Forensic model on prevention of violence at the interface to general psychiatry: the forensic psychiatric liaison service}

\begin{abstract}
Even though a subgroup of mentally ill people is at a heightened risk for committing violence and patients from forensic clinics usually have a general psychiatric history, to date preventive treatment modalities at the interface between forensic and general psychiatry are rare. This article reviews different possibilities for interdisciplinary collaboration and describes the services offered by the forensic psychiatric liaison service of the special unit for forensic assessment and risk management (FFA) of the Clinic for Forensic Psychiatry (KFP) at the Psychiatric University Hospital Zurich (PUK).
\end{abstract}

Keywords Risk management $\cdot$ Forensic expertise $\cdot$ Preventive outpatient clinic $\cdot$ Risk factors $\cdot$ Support of general psychiatry

\section{Einleitung}

Im allgemeinpsychiatrischen Setting werden seit Jahren Betten abgebaut (Chow und Priebe 2016). Das ambulante Angebot wird erweitert, insbesondere aufsuchende Be-

Dr. med. Dipl.-Jur. Catharina Schmidt catharina.schmidt@pukzh.ch

1 Klinik für Forensische Psychiatrie, Psychiatrische Universitätsklinik Zürich, Lenggstraße 31, 8032 Zürich, Schweiz

2 Bezirkskrankenhaus Straubing, Lerchenhaid 32, 94315 Straubing, Deutschland handlungsmodelle gewinnen an Bedeutung (Munz et al. 2011; Lambert et al. 2014). Gleichzeitig stellt eine niedrige Anzahl von Zwangsbehandlungen ein Qualitätsmerkmal allgemeinpsychiatrischer Behandlungen dar (Großimlinghaus et al. 2017). Im forensisch-psychiatrischen Bereich ist die Entwicklung gegenläufig, d.h., die Bettenzahlen steigen kontinuierlich. Im forensischen Kontext scheint auch die Akzeptanz belastender Zwangsmaßnahmen ungleich größer zu sein (Tribolet-Hardy und Habermeyer 2016; Brackmann et al. 2020).

Seit Jahren gibt es Hinweise darauf, dass eine Untergruppe psychisch kranker Personen, insbesondere Betroffene mit einer Erkrankung aus dem schizophrenen Formenkreis, von den skizzierten Entwicklungen in der Allgemeinpsych- 
iatrie nicht zu profitieren scheint und für die steigenden Zahlen im Maßregelvollzug mitverantwortlich ist (Hodgins et al. 2006). Personen, die an einer Schizophrenie leiden, haben infolge ihrer Erkrankung ohnehin eine belastete Risikoprognose (Fazel et al. 2009). Obwohl sie im Vorfeld ihrer Unterbringungsdelikte zumeist stationär allgemeinpsychiatrisch behandelt wurden (Habermeyer et al. 2010), scheinen sie häufig trotz fortbestehender schwerer Symptombelastung aus der Allgemeinpsychiatrie entlassen zu werden (Lincoln et al. 2006). Gewaltassoziiertes Fehlverhalten, aber auch mangelhafte Compliance mit häufigen Behandlungsabbrüchen sowie ein zusätzlicher Substanzmissbrauch belasten ihre Risikoprognose zusätzlich und führen zu erschwerten Behandlungsbedingungen (Seliger und Kröber 2008). Auch lässt sich nicht jeder allgemeinpsychiatrische Patient problemlos in vorhandene Nachsorgestrukturen einbinden. Somit bleibt eine Risikoklientel trotz aufwendiger Bemühungen nur schwer erreichbar (Lincoln et al. 2006).

Dennoch wird vermutet, dass intensivere präventive Maßnahmen mittels leitlinienorientierter, sozialpsychiatrischer Behandlung und Fokus auf modifizierbare Risikofaktoren ein von diesen Personen ausgehendes Gewaltrisiko senken können (Nitschke et al. 2011; Wolf et al. 2017). Da zusätzlich eine geringere Einbindung in ein psychiatrisches Behandlungssystem ein Risikomerkmal für eine spätere Maßregelunterbringung darstellt (Piontek et al. 2013), zielen Modelle zur Prävention von Gewalttaten psychisch kranker Menschen darauf ab, diese Klientel zu erreichen. Dies ist die Rationale für entsprechende, präventiv ausgerichtete Angebote:

Dabei stellen einige Modelle ein eigenständiges Behandlungsangebot für gewaltbereite Personen bereit, das mittels geeigneter therapeutischer Interventionen versucht, dynamische Risikofaktoren zu reduzieren. Andere Ansätze sind auf die Beratung und Unterstützung der allgemeinpsychiatrischen Kliniken bzw. der gemeindenahen Patientenversorgung ausgerichtet, indem sie beispielsweise die Durchführung von Risk assessments und eine Unterstützung im Risk management anbieten. Denn die Identifikation von Risikofaktoren wie auch die Etablierung eines auf den Einzelfall abgestimmten Fallkonzepts und eines Risikomanagements sind in der forensischen Psychiatrie anerkannte Standardprozedere, welche auch im allgemeinpsychiatrischen Setting genutzt werden können (Hodgins 2006; Seliger und Kröber 2008). Die Anwendung von kriminalprognostischen Instrumenten benötigt allerdings Zeit, die im stationären Behandlungssetting häufig fehlt, und setzt in der Regel Spezialwissen voraus, das im Rahmen von Schulungen erworben werden muss.

Beiden Ansätzen ist gemein, dass gewaltassoziierte Verhaltensweisen, die eng mit einer psychischen Erkrankung in Verbindung stehen, in einem psychiatrisch-psychotherapeutischen risikofokussierten Kontext (zumeist außerhalb juristisch verfügter Behandlungen) adressiert werden. Sie dienen u. a. der Verhinderung von kostspieligen forensischpsychiatrischen Unterbringungen und einem effektiven Opferschutz. Nachfolgend werden Beispiele aus Deutschland und der Schweiz vorgestellt.

\section{Eigenständige Behandlungsangebote}

\section{Präventionsambulanz Ansbach}

Orientiert am „Hamburger Modell“, einem Ansatz zur integrativen Versorgung von Menschen mit psychotischen Störungen (Lambert et al. 2014), werden in der Präventionsambulanz der Klinik für Forensische Psychiatrie des Bezirksklinikums Ansbach (Nitschke et al. 2020a) seit 2012 Patienten mit Erkrankungen aus dem schizophrenen Formenkreis und/oder schweren Persönlichkeitsstörungen behandelt, bei denen im Falle strafrechtlich relevanten Verhaltens die Voraussetzungen für eine Behandlung im Maßregelvollzug gemäß § 63 StGB wahrscheinlich erfüllt wären.

Diesen Personen wird eine multiprofessionelle, umfassende, störungsorientierte Behandlung mit einem besonderen Fokus auf ein forensisches Risikomanagement angeboten. Durch eine enge Zusammenarbeit mit der Allgemeinpsychiatrie, aber auch niedergelassenen Ärzten, professionellen Betreuungspersonen und Angehörigen wird eine settingübergreifende Behandlungskontinuität sichergestellt: Sind z.B. zwischenzeitlich stationäre allgemeinpsychiatrische Aufnahmen der Patienten aus der Präventionsambulanz erforderlich, suchen die Mitarbeitenden der Präventionsambulanz während dieser Aufenthalte ihre Patienten auf und sind insbesondere bei der Entlassplanung Ansprechpartner.

Die wissenschaftliche Ausrichtung der Präventionsambulanz erlaubt eine Evaluation der Wirksamkeit der präventiven Behandlung. In einer 24-monatigen prospektiven Longitudinalstudie wurde die Behandlung in der Präventionsambulanz $(n=70)$ mit einer Kontrollgruppe, die eine herkömmliche psychiatrische Betreuung erhielt $(n=52)$, verglichen (Nitschke et al. 2020b): Das durch die R-Skala des HCR-20 operationalisierte dynamische Risiko (Douglas et al. 2013) fiel in der Behandlungsgruppe im Vergleich zur Kontrollgruppe im Zeitverlauf signifikant stärker ab. Trotz hoher Effektstärke (Morris $\mathrm{d}=-0,80$ ) konnte jedoch eine Konfundierung durch inhärente Gruppenunterschiede aufgrund der relativ kleinen Stichprobe nicht ausgeschlossen werden. Innerhalb der Risiko-Items des HCR-20 zeigten sich bei den Items Stress (Cohens $d=0,76)$ und destabilisierende Faktoren (Cohens $\mathrm{d}=-0,61$ ) die stärksten Effekte in der Behandlungs- gegenüber der Kontrollgruppe.

Mittlerweile fand das Konzept der Präventionsambulanz auch Eingang in das Psychisch-Kranken-Hilfe-Gesetz des 
Landes Bayern (Art. 51 BayPsychKHG, Bayerische Staatsregierung (2018)) und soll landesweit etabliert werden.

Art. 51 Präventionsstellen Das Zentrum Bayern Familie und Soziales wirkt darauf hin, dass an Einrichtungen für forensische Psychiatrie ein bedarfsgerechtes Angebot an Vorsorgemaßnahmen für psychisch kranke Menschen geschaffen wird, bei denen aufgrund der Art und Schwere ihrer Erkrankung ein stark erhöhtes Risiko für Handlungen besteht, die eine Unterbringung nach § 63 StGB zur Folge haben könnten.

Das Angebot der Präventionsambulanz stellt ein forensisch-psychiatrisches Substitut für eine allgemeinpsychiatrische ambulante Behandlung dar. Die Unterhaltung eines solchen umfassenden Behandlungsangebots benötigt einige zeitliche und kostenintensive Ressourcen, die jedoch angesichts der erwarteten Kostenersparnisse durch Abwendung von Maßregelbehandlungen schnell refinanziert sind (Nitschke et al. 2018). Allerdings bedingt das eigenständige Behandlungsangebot, dass eine problematische Patientengruppe aus dem allgemeinpsychiatrischen Behandlungssystem ausgegliedert und aufgrund ihrer ungünstigen Risikoprognose bereits vor Begehung gravierender Delikte dem forensischen Fachgebiet zugeordnet wird. Eine solche Entwicklung wird durchaus auch kritisch betrachtet: Turner und Salter (2005) präferieren den Verbleib von risikoorientierten Behandlungen im Rahmen klinischer Routinebehandlungen, denn die Vorstellung, dass ein außenstehender Experte Risiken effektiver managen könne, sei nicht bewiesen.

\section{Kooperationsprojekte mit allgemeinpsychiatrischen Angeboten}

\section{Ärger-Wut-Aggressions-Gruppe}

Auch psychotherapeutische Angebote wurden in den letzten Jahren geschaffen, um gezielte Gewaltprävention zu betreiben. Wie im ausführlichen Artikel in dieser Ausgabe berichtet, werden in der Ärger-Wut-Aggressions(AeWA)Gruppe (Schröter et al. 2021) persönlichkeitsgestörte Männer mit einer Störung der Impulskontrolle behandelt. Das Angebot trägt dem Umstand Rechnung, dass gezielte psychotherapeutische Programme wie etwa der dialektisch-behaviorale Ansatz zur Behandlung der Borderline-Persönlichkeitsstörung (Linehan et al. 1991) in erster Linie auf eine weibliche Klientel ausgerichtet sind und spezifische therapeutische Programme für aggressive Verhaltensweisen für männliche Personen mit Persönlichkeitsstörung fehlen. In einem kognitiv-behavioral ausgerichteten Modulsystem werden gezielt soziale Kompetenzen verbessert und sozial angemessenes Verhalten verstärkt, um impulsives wie aggressives Verhalten zu verringern.

\section{Forensische Behandlungen und Beratungen im Zentrum für Abhängigkeitserkrankungen der Psychiatrischen Universitätsklinik Zürich}

Hinsichtlich der Behandlung von Personen mit Substanzgebrauchsstörungen und einer in diesem Zusammenhang möglichen Delinquenz liegt ebenfalls eine enge Zusammenarbeit mit einem forensischen Dienst nahe (Höfer et al. 2019): Die Strukturen des ambulanten Suchtbehandlungszentrums (einschl. der Opioidabgabestelle) werden genutzt, um ambulante Suchtmaßnahmen durchzuführen. Durch die Anwesenheit eines forensischen Dienstes vor Ort ist zudem sichergestellt, dass auch bei Patienten ohne Maßnahmenbehandlung auf forensische Expertise zugegriffen werden kann. Gemeinsame Teamsitzungen, das Angebot einer 24-h-Notfallbetreuung und die Möglichkeit der Behandlung von somatischen Komorbiditäten erlauben eine umfassende Behandlung einer häufig herausfordernden Patientenklientel.

\section{Forensisch-psychiatrische Liaison- bzw. Konsilmodelle}

Neben den skizzierten Behandlungsmodellen existieren auch präventive Ansätze, die forensisch-psychiatrische Beratungsleistungen, u.a. für allgemeinpsychiatrisch tätige Behandler, vorsehen. Dadurch kann die gemeindenahe Patientenversorgung ganz unabhängig von einem formaljuristischen Setting von einem forensischen Beratungsangebot profitieren:

In den Niederlanden (Polhuis et al. 2017) stehen den gemeindenahen psychiatrischen Versorgungsdiensten forensische Experten zur Verfügung, die nach individueller Fallanalyse und Risk assessment Empfehlungen aussprechen und ggf. auch forensische „key partner" (forensische Behandlungsprogramme, Polizei etc.) kontaktieren. In Kanada werden durch die „Clinique d'évaluation du risque du violence“ des Institut national de psychiatrie légale PhilippePinel (Québec) (2021) behandelnden Einrichtungen forensische Beratungsleistungen angeboten, die darauf abzielen, die Behandlung wiederholt gewalttätig gewordener Patienten mit einer Achse-I-Störung, deren Gefährlichkeit schwer einschätzbar oder schwer behandelbar ist, zu verbessern. Ausschlusskriterien für das Beratungsangebot stellen geistige Behinderungen, Demenzen und organische Verhaltensstörungen dar. Notfallkonsultationen für Betroffene ohne psychiatrische Betreuung werden nicht vorgenommen.

In England existieren einige durch den National Health Service unterstützte forensisch-psychiatrische Konsil- bzw. 
Liaisonmodelle mit aufsuchendem Charakter (Forensic Outreach Liaison Services [FOLS] (2021)), die in einem multiprofessionellen Team die gemeindenahe Patientenversorgung v. a in der Nachsorge forensisch entlassener Patienten, aber auch mittels ausgeweitetem Risikomanagement unterstützen kann. Das Angebot reicht dabei von regelmäßigen Besprechungen in den betreuenden Einrichtungen vor Ort bis zur Durchführung von Risk assessments und Supervisionsangeboten. Einige Dienste adressieren primär Betroffene mit Autismus und Intelligenzminderungen, die ein Risiko für Gewalttaten haben und ggf. bereits mit strafrechtlich relevanten Verhaltensweisen in Erscheinung getreten sind.

Natarajan et al. (2012) beschreiben das Wolverhampton Mental Health Services Forensic Liaison Scheme (WMHSFLS), welches eine enge Zusammenarbeit zwischen einer erwachsenenpsychiatrischen Klinik und dem Hatherton Centre (einer forensisch geführten, gesicherten Klinik in Stafford) vorsieht: Die Kliniker des forensischen Dienstes sind auch Mitglieder eines multiprofessionellen Klinikteams am Hatherton Centre, das für die stationäre Versorgung von Patienten aus Wolverhampton zuständig ist. Wöchentlich finden Sitzungen mit einem beratenden forensischen Psychiater und einer forensischen Krankenschwester statt. Auswertungen der Aktivitäten des Liaisonprojektes haben ergeben, dass der überwiegende Anteil zugewiesener Patienten an einer psychotischen Episode litt. Die Mitarbeitenden des Wolverhampton Mental Health Service hätten insbesondere das Angebot der Risikoeinschätzung und des Risikomanagements als hilfreich empfunden. Die Autoren formulierten Kriterien, die einen forensisch-psychiatrischen Konsil- bzw. Liaisondienst auszeichnen (Tab. 1).

\section{Der Forensisch-psychiatrischer Konsildienst des Kantons Zürich}

\section{Entwicklung}

Bis zum Jahr 2012 gab es auch im Kanton Zürich keine definierten Informations- und Abklärungsprozesse oder Behandlungspfade an der Schnittstelle zwischen Allgemein-

Tab. 1 Vorteile der Arbeit in einem Liaisonmodell gemäß Natarajan et al. (2012)

Kontinuität der Betreuung zwischen psychiatrischen Diensten und dem Strafjustizsystem

Schneller Zugang zum Expertenrat in Bezug auf Risikobewertung und -management

Koordination von Aufnahmen und Entlassungen

Produktive Arbeitsbeziehungen zwischen forensischen und gemeindenahen psychiatrischen Diensten

Unterstützung der gemeindenahen Patientenversorgung im komplexen Fallmanagement, bei Risikobewertung und -management

Gesundheitliche und wirtschaftliche Vorteile durch Leistungsintegration/-ausrichtung psychiatrie und forensischer Psychiatrie. Dies hatte zur Folge, dass die psychiatrischen Akutkliniken beim Umgang mit gewaltbereiten Personen nicht systematisch auf vorhandenes forensisch-psychiatrisches Expertenwissen zur Abschätzung des Gefährdungspotenzials zugreifen konnten, dass ihnen kein spezialisiertes Wissen über evtl. einsetzbare strafrechtliche Instrumente zur Verfügung stand, und dass bei entsprechendem Bedarf keine Entlastungsmöglichkeit durch die Verlegung einzelner Patienten zur gezielten Krisenintervention in einem gesicherten Umfeld angeboten werden konnte.

Zur Verbesserung der Situation beim Umgang mit gewaltbereiten Personen hatte der Regierungsrat am 31.08.2011 mit Regierungsratsbeschluss (RRB) Nr. 1048 (2011) die Gesundheitsdirektion beauftragt, eine mobile Triage-Equipe zur raschen Beurteilung und sachgerechten Unterbringung und Behandlung von gewaltbereiten Personen $\mathrm{zu}$ schaffen und die notwendigen Maßnahmen für den Umgang mit gewaltbereiten Personen durch konsiliarische Beratung und Unterstützung in der Akutpsychiatrie umzusetzen. Kernaufgaben der Triage-Equipe sollten dabei das Durchführen von Risikoeinschätzungen, das Abgeben von Empfehlungen für das weitere Vorgehen, das Sicherstellen einer guten Vernetzung der zuständigen Stellen, das Festlegen klarer Abläufe und die Dokumentation der Fälle zwecks besserer Beurteilung des Versorgungsbedarfs sein. Im Bedarfsfall, d.h. bei fehlender Führbarkeit in der Allgemeinpsychiatrie, werden auch Verlegungen zur Krisenintervention in eine forensisch geführte Einrichtung organisiert. Es lag nahe, die Forensisch-Psychiatrische Abklärungs- und Beratungsstelle in der Klinik für Forensische Psychiatrie (KFP) der Psychiatrischen Universitätsklinik Zürich (PUK) anzusiedeln.

Mittlerweile wurde das Beratungsangebot in die Fachstelle Forensic Assessment \& Risk Management (FFA) der KFP der PUK integriert. Während die Fachstelle zuvor ausschließlich fachfremde Stellen (Polizei und Staatsanwaltschaft) im Rahmen ihrer klar definierten, gesetzlichen Aufträge bzgl. des Risikomanagements gewaltbereiter Personen unterstützte (Beyli-Helmy et al. 2020), kam mit der Beratung der akutpsychiatrischen Versorgungskliniken des Kantons Zürich ein neuer Auftraggeber mit psychiatrischpsychologisch tätigen Behandlern dazu. Die Unterstützung der Kliniken im Risikomanagement ihrer Patienten erfolgt dann, wenn dies zur Erfüllung ihres allgemeinen Leistungsauftrags relevant ist: etwa bei der Einschätzung der Fremdgefährlichkeit von Patienten z. B. in Zusammenhang mit fürsorgerischen Unterbringungen (schweizerisches Pendant zur Unterbringung nach PsychKG) oder bei Fragen im Zusammenhang mit der Behandlung fremdgefährlicher Aspekte wie etwa der Einschätzung von Gewaltfantasien.

Die Entwicklung des Konsildienstes wird durch regelmäßige Sitzungen einer interdisziplinären Fachkommission 
Abb. 1 Übersicht der Abläufe

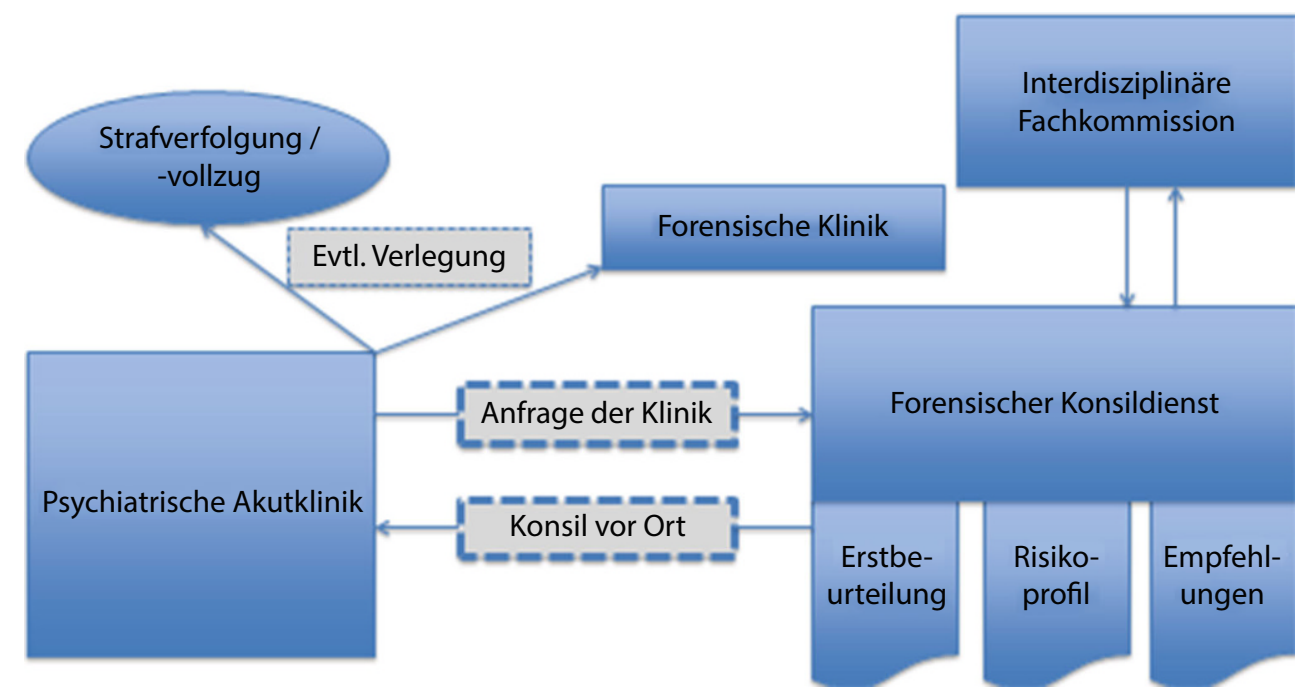

(Fachkommission zur Behandlung gewaltbereiter Personen mit Vertretern der Gesundheitsdirektion, der Staatsanwaltschaft, Vertretern der beratenden Kliniken und der Kindesund Erwachsenenschutzbehörde) begleitet. Damit wurde eine übergeordnete Instanz zur Qualitätssicherung geschaffen, die durch Einzelfälle aufgedeckte unbefriedigende Istzustände, Unsicherheiten und Fallstricke im System bzw. unter den beteiligten Fachleuten diskutiert, Lösungsansätze erarbeitet und implementiert. Die Ausrichtung der Fachkommission ist strategischer Natur; sie ist nicht operativ/ beratend in Fallbearbeitungen beteiligt. Die Fachkommission tagt vierteljährlich; die Sitzungen und die Arbeit der Fachkommission werden von der Klinik für Forensische Psychiatrie koordiniert (Abb. 1).

\section{Leistungsangebot}

\section{Telefonische Beratungen}

Die akutpsychiatrischen Versorgungskliniken des Kantons Zürich können niederschwellig und vorerst anonymisiert forensische Fragestellungen an die FFA richten und sich zur Risikoeinschätzung, zum Fallmanagement oder aber auch zu diagnostischen Problemstellungen und Behandlungsoptionen umfassend beraten lassen.

\section{Fallbesprechungen/Supervision in den Kliniken}

Seit 2017 erfolgen in den akutpsychiatrischen Versorgungskliniken des Kantons regelmäßig forensische Fallbesprechungen und Supervisionen. Die Fallbesprechungen erfolgen entweder mit Einwilligung der betroffenen Personen oder anonymisiert. Die Kliniken können im Vorfeld Problembereiche bzw. Fälle benennen, die mit besonderem
Fokus auf forensische Risikoeinschätzungen diskutiert werden.

\section{Forensische Konsile im stationären Klinikbereich}

Die akutpsychiatrischen Versorgungskliniken des Kantons Zürich können im Rahmen forensischer Konsile innert weniger Arbeitstage Risikoeinschätzungen potenziell gewaltbereiter Personen vornehmen und sich hinsichtlich weiterer Maßnahmen zur risikosenkenden Behandlung beraten lassen. Sollte der Patient nicht in eine Untersuchung einwilligen, kann eine Risikoeinschätzung auf der Grundlage zuvor anonymisierter Akten durchgeführt werden.

\section{Ablauf}

Die Kontaktaufnahme zum forensischen Konsildienst erfolgt nach oberärztlicher Indikationsstellung hinsichtlich eines relevanten Konsilanlasses. Eine initiale Aufklärung der betroffenen Patienten über Hintergrund, Ablauf und mögliche Empfehlungen eines forensischen Konsils wird durch die anmeldenden Oberärzte durchgeführt. Die Einwilligung in die Untersuchung und Dokumentation der Freiwilligkeit der Teilnahme wird schriftlich festgehalten. Liegt nach ausreichender Bedenkzeit kein Einverständnis vor oder ist die betroffene Person diesbezüglich nicht urteilsfähig, erfolgt keine konsiliarische Untersuchung. In diesen Fällen ist jedoch eine Beratung zur Risikoeinschätzung anhand anonymisierter Patienteninformationen möglich. Die Reaktionszeit bezüglich konsiliarischer Anfragen beträgt in der Regel $24 \mathrm{~h}$.

Die Patienten werden auf der Station zumeist einmalig forensisch-konsiliarisch untersucht. Die Einschätzung erfolgt - je nach dem allenfalls bereits erfolgten gewalttätigen Vorfall - basierend auf gängigen Prognoseinstrumenten, z. B dem HCR-20 V3 (Douglas et al. 2013). Im 
Anschluss an die Untersuchung wird in der Regel innerhalb von $72 \mathrm{~h}$ ein schriftlicher Konsilbericht erstellt, der die vom Auftraggeber gestellten Fragen beantwortet und Maßnahmen zur Gewaltprävention formuliert. Wie in allen anderen Bereichen der ärztlichen Tätigkeit unterliegen die Konsilärzte der Schweigepflicht. Eine Weitergabe erstellter Konsilberichte erfolgt nicht bzw. nur nach ausdrücklich erteiltem Einverständnis der untersuchten Personen.

Die Anlässe für Konsiluntersuchungen variieren. Nicht immer geht es um eine konkrete Risikoeinschätzung, wie sie sich beispielsweise im Zusammenhang mit der Ausführungsgefahr einer Drohung ergibt. Gelegentlich sind auch diagnostische Einschätzungen und Behandlungsstrategien aus forensisch-psychiatrischer Perspektive gefragt, wie etwa im Falle grenzverletzenden Verhaltens und einer sich in diesem Zusammenhang ergebenden medikamentösen Indikation. Entsprechende Empfehlungen beziehen sich auf die Behandlung mit dem Ziel der Reduktion aggressiven Verhaltens, etwa durch Optimierung medikamentöser Strategien, Vermittlung von forensisch erfahrenen Weiterbehandlern, Behandlungsprogrammen und Vorschläge geeigneter Wohnheimplätze. Gelegentlich werden auch Verlegungen in eine forensisch-geführte Einrichtung angefragt. In diesen Fällen unterstützen die Konsilärzte bei der Organisation bzw. Umsetzung.

Das vorgehaltene Angebot wird regelmäßig genutzt, mit bis ca. 30 Einschätzungen/Jahr. Derzeit wird eine Auswertung der erhobenen Konsildaten vorgenommen, die dann eine exakte Beschreibung der untersuchten Stichprobe ermöglicht. Schon jetzt kann festgehalten werden, dass ca. die Hälfte der untersuchten Personen an einer Erkrankung aus dem schizophrenen Formenkreis litt.

\section{Mögliche Ausweitung des Leistungsangebots}

Im Rahmen der Konsiltätigkeiten war es bisher nicht möglich, Risikopatienten nach ihrem Klinikaustritt weiterhin forensisch zu betreuen bzw. die mit der ambulanten Behandlung betrauten Institutionen zu beraten, um ein settingübergreifendes, kontinuierliches Risikomanagement zu unterstützen.

Sowohl auf den allgemeinpsychiatrischen Akutstationen als auch auf den Therapiestationen und im ambulanten Kliniksetting sollte für die Behandlungsteams die Möglichkeit bestehen, sich umfassend bei der Risikoeinschätzung, der Behandlung und dem Management von Risikopatienten beraten zu lassen. Erste Maßnahme zum Ausbau des bestehenden unterstützenden Angebots stellt daher die regelmäßige Anwesenheit einer forensischen Fachperson in den ambulanten und stationären Einrichtungen der Versorgungskliniken dar, um die Hemmschwellen in der Zusammenarbeit zu reduzieren und systematisch Zugriff auf forensisches Wissen sicherzustellen (,,forensische Sprechstunde“).
Eine begleitende, fachspezifische, zusätzliche Behandlung eines Patienten durch Vertreter zusätzlicher Subdisziplinen ist im medizinischen Setting im Rahmen einer sog. Liaisonbehandlung bereits etabliert. In der Psychiatrie gibt es solche Modelle z.B. im Bereich der Alterspsychiatrie bzw. im Rahmen von Wohnheimkonzepten (Laireiter et al. 2015) oder aber, wie oben beschrieben, im Bereich der Abhängigkeitserkrankungen (Höfer et al. 2019). Mit der Etablierung eines forensischen Liaisonmodells im allgemeinpsychiatrischen Setting kann eine begleitende forensische Behandlung von Risikopatienten ermöglicht werden. Auf diese Weise ist sichergestellt, dass bestehende Risikofaktoren für gewalttätiges Verhalten regelmäßig evaluiert und krisenhafte Verläufe schneller entdeckt werden.

Die Vorzüge von forensischen Liaisonmodellen bestehen in einer settingübergreifenden Behandlungskontinuität zwischen Behandlungseinrichtungen, aber auch strafrechtlicher juristisch verfügter Maßnahmen, schnellem Zugang zu Risikoeinschätzungen und forensischen Beratungsleistungen (Natarajan et al. 2012). Zusätzlich bestehen ein Vorteil durch eine Supervision von Aufnahmen, Entlassungen und Behandlungen sowie ein Produktivitätsvorteil zwischen forensischer Psychiatrie und der gemeindenahen Versorgung. Denn nicht nur in der forensischen Psychiatrie fehlen personelle Ressourcen. Auch in der Allgemeinpsychiatrie wird die tägliche Arbeit durch personelle Engpässe erschwert.

Die Nutzung allgemeinpsychiatrischer Strukturen für die Umsetzung eines gewaltpräventiven Ansatzes stellt, im Gegensatz zu einem eigenständigen Behandlungsangebot, eine kostengünstige Alternative dar. Einschränkend ist anzumerken, dass ein forensisches Liaison- bzw. Konsilangebot ein vorbestehendes Behandlungsverhältnis und die Bereitschaft der Behandler voraussetzt, den Liaisondienst in Anspruch zu nehmen.

\section{Fazit}

Gewaltprävention an der Schnittstelle zur Allgemeinpsychiatrie kann über unterschiedliche Ansätze verfolgt werden. Sie adressieren eine Patientenklientel mit einem hohen Risikoprofil für interpersonelle Gewalttaten, deren Behandlung in der allgemeinpsychiatrischen Praxis aufgrund gewaltassoziierter Verhaltensweisen herausfordernd ist. Das Potenzial gewaltpräventiver Maßnahmen v. a für eine Risikoklientel von Patienten mit Erkrankungen aus dem schizophrenen Formenkreis in der Allgemeinpsychiatrie ist beträchtlich, denn es bestehen ausreichende Gelegenheiten für die Umsetzung präventiv wirksamer Maßnahmen angesichts einer langen allgemeinpsychiatrischen Gesamtbehandlungsdauer vor dem Unterbringungsdelikt (Habermeyer et al. 2010) und eines durchschnittlichen Zeitraums zwischen der ersten sta- 
tionären Behandlung und der Begehung eines Unterbringungsdelikts von ca. 7 Jahren (Piontek et al. 2013).

Die Grundlagen gewaltpräventiv-psychiatrischen Denkens fanden mittlerweile auch auf juristisch-politischer Ebene hinsichtlich des Umgangs bezüglich psychisch kranker Menschen, die ein erhöhtes Gewaltrisiko aufweisen, Berücksichtigung: Gesetzliche Grundlagen für die Umsetzung präventiver Angebote an der Schnittstelle zur Allgemeinpsychiatrie wurden geschaffen und Modellprojekte entsprechend gefördert. Denn Aggression und gewaltassoziierte Verhaltensweisen können eng mit einer psychiatrischen Grunderkrankung verknüpft sein.

Allerdings werden die an der Risikoeinschätzung der Betroffenen ausgerichtete Behandlung und die Integration eines Risk management in das allgemeinpsychiatrische Setting auch kontrovers diskutiert: Kritische Stimmen sehen die Gefahr einer Benachteiligung von Patienten mit belasteter Risikoprognose und eine zusätzliche Stigmatisierung bei nur eingeschränktem prädiktivem Wert einer risikoprognostischen Einschätzung (Zinkler, 2009; Wössner, 2018).

Dabei geht es letztlich darum, Personen zu identifizieren, bei denen ein besonderer Hilfebedarf vorliegt. Ihre fachgerechte Behandlung weicht nicht von vorgegebenen Standards der allgemeinpsychiatrischen Versorgung ab. Nur ist zumeist der Zugang der beschriebenen häufig herausfordernden und belasteten Risikogruppe zum Behandlungssystem infolge von problematischen, gewaltassoziierten Verhaltensweisen, Behandlungsabbrüchen und Substanzkonsum erschwert und erfordert intensivere Behandlungsbemühungen. Denn der weitere Verlauf hängt entscheidend vom Umfang und von der Qualität der stationären und ambulanten engagierten Betreuungsangebote ab. Im Fokus der Behandlung stehen zumeist effektive medikamentöse Behandlungsstrategien, einschließlich der Absicherung ihrer Behandlungskontrolle, und sozialpsychiatrische Maßnahmen, die einer sozialen Desintegration entgegenwirken. Die Behandlung beinhaltet engmaschige Kontakte und verbindliche Absprachen bezüglich der Interventionsstrategien bei Krisen unter Einbezug des sozialen Umfelds, welches häufig selbst Opfer gewaltassoziierter Verhaltensweisen wird. Dabei steht außer Frage, dass das ambulante und stationäre Kliniksetting für Patienten den Schutzraum bieten soll, um bei akuten Krisen und/ oder fortwährendem psychischem Leidensdruck Entlastung zu erfahren. Die betroffenen Personen sollen tragfähige Strategien für den Umgang mit gewaltassoziierten Verhaltensweisen erarbeiten, ohne Gefahr zu laufen, aufgrund ihrer Problemstellungen wie z.B. Aggression stigmatisiert zu werden.

Die hier skizzierten Modelle zeigen auf, dass ein Bedarf an forensischer Expertise auch außerhalb juristisch verfügter Behandlungssettings besteht und ein solches Angebot für die häufig selbst durch aggressives Verhalten und die sich daraus ergebenden Konsequenzen belasteten Personen auf freiwilliger Basis in Anspruch genommen wird. Sie stellen einen Ansatz dar, risikoprognostisches Denken zur Gewaltprävention einzusetzen, und bieten Anlass, die vielerorts strikt eingehaltene Trennung allgemeinpsychiatrischen und forensisch-psychiatrischen Handelns zu überdenken, denn beide Bereiche können und sollten sinnvollerweise zusammenarbeiten. Eine ohnehin häufig anzutreffende räumliche Nähe allgemeinpsychiatrischer und forensischer Behandlungsangebote erleichtert die Zusammenarbeit und birgt, auch im Hinblick auf eine Nutzung gemeinsamer räumlicher und personeller Strukturen, enormes wirtschaftliches Potenzial. Nicht zuletzt stellen die enge Zusammenarbeit der Einrichtungen und die Präsenz der forensischen Psychiatrie vor Ort auch eine Chance dar, interessierten Nachwuchs für das forensische Fachgebiet zu begeistern.

Funding Open access funding provided by University of Zurich

Interessenkonflikt C. Schmidt, J. Nitschke und E. Habermeyer geben an, dass kein Interessenkonflikt besteht.

Open Access Dieser Artikel wird unter der Creative Commons Namensnennung 4.0 International Lizenz veröffentlicht, welche die Nutzung, Vervielfältigung, Bearbeitung, Verbreitung und Wiedergabe in jeglichem Medium und Format erlaubt, sofern Sie den/die ursprünglichen Autor(en) und die Quelle ordnungsgemäß nennen, einen Link zur Creative Commons Lizenz beifügen und angeben, ob Änderungen vorgenommen wurden.

Die in diesem Artikel enthaltenen Bilder und sonstiges Drittmaterial unterliegen ebenfalls der genannten Creative Commons Lizenz, sofern sich aus der Abbildungslegende nichts anderes ergibt. Sofern das betreffende Material nicht unter der genannten Creative Commons Lizenz steht und die betreffende Handlung nicht nach gesetzlichen Vorschriften erlaubt ist, ist für die oben aufgeführten Weiterverwendungen des Materials die Einwilligung des jeweiligen Rechteinhabers einzuholen.

Weitere Details zur Lizenz entnehmen Sie bitte der Lizenzinformation auf http://creativecommons.org/licenses/by/4.0/deed.de.

\section{Literatur}

Bayerische Staatsregierung (2018) Bayerisches Psychisch-KrankenHilfe-Gesetz (BayPsychKHG) vom 24. Juli 2018 (2128-2-A/G). Gesetz Verordnungsbl 14:583

Beyli-Helmy M, Habermeyer E, Guldimann A (2020) Was kann die Forensische Psychologie und Psychiatrie im Bedrohungsmanagement beitragen? Erkenntnisse aus der interdisziplinären Zusammenarbeit im Kanton Zürich. RPsych 6(3):357-370

Brackmann N, Lau S, Mokros A, Habermeyer E (2020) Aims to reduce coercive measures in forensic inpatient treatment: a 9-year observational study. Front Psychiatry 11:465

Chow WS, Priebe S (2016) How has the extent of institutional mental healthcare changed in Western Europe? Analysis of data since 1990. BMJ Open 6(4):e10188

Douglas KS, Hart SD, Webster CD, Belfrage H (2013) HCR-20 assessing risk for violence V3 (Burnaby Simon Fraser University, Mental Health, Law, and Policy Institute)

Fazel S, Gulati G, Linsell L, Geddes JR, Grann M (2009) Schizophrenia and violence: systematic review and meta-analysis. PLoS Med 6(8):e1000120 
Forensic outreach liaison service (FOLS) (2021). https://www.south westyorkshire.nhs.uk/services/forensic-outreach-liaisonservice-fols/. Zugegriffen: 3. Juni 2021

Großimlinghaus I, Hauth I, Falkai P, Janssen B, Deister A, Meyer-Lindenberg A, Roth-Sackenheim C, Schneider F, Wobrock T, Zeidler R, Gaebel W (2017) Aktuelle Empfehlungen der DGPPN für Schizophrenie-Qualitätsindikatoren. Nervenarzt 88(7):779-786

Habermeyer E, Wolff R, Gillner M, Strohm R, Kutscher S (2010) Patienten mit schizophrenen Störungen im psychiatrischen Maßregelvollzug. Nervenarzt 81(9):1117-1124

Hodgins S (2006) Gewalt und Kriminalität bei psychisch Kranken. Neue Erkenntnisse erfordern neue Lösungen. Neuropsychiatrie 20:7-14

Hodgins S, Müller-Isberner R, Allaire JF (2006) Attempting to understand the increase in the numbers of forensic beds in Europe: a multi-site study of patients in forensic and general psychiatric services. Int J Forensic Ment Health 5(2):173-184

Höfer F, Caflisch C, Herdener M, Habermeyer E (2019) Ambulante Suchtmaßnahmen in der Schweiz: eine Alternative zum deutschen geschlossenen Maßregelvollzug nach § 64? Forens Psychiatr Psychol Kriminol 13:272-281

Institut national de psychiatrie légale Philippe-Pinel (2021) https:// pinel.qc.ca/clinique-devaluation-du-risque-de-violence/. Zugegriffen: 3. Juni 2021

Laireiter AR, Baumann U, Messer R (2015) Gerontopsychologie in Einrichtungen der Seniorenpflege. Alterspsychotherapie und klinische Gerontopsychologie. Springer, Berlin, Heidelberg, New York, S 289-313

Lambert M, Schöttle D, Bock T, Schulte-Markwort M, Naber D, Karow A (2014) Hamburger Modell der integrierten Versorgung von schwer erkrankten Psychosepatienten. Psychotherapeut 59(2):95-99

Lincoln T, Sheilagh H, Jöckel D, Freese R, Born P, Eucker SH, Schmidt P, Gretenkord L, Müller-Isberner R (2006) Forensische Patienten und Patienten der Allgemeinpsychiatrie. Nervenarzt 77:576-586

Linehan MM, Armstrong HE, Suarez A, Allmon D, Heard HL (1991) Cognitive-behavioral treatment of chronically parasuicidal borderline patients. Arch Gen Psychiatry 48(12):1060-1064

Munz I, Ott M, Jahn H, Rauscher A, Jäger M, Kilian R, Frasch K (2011) Vergleich stationär-psychiatrischer Routinebehandlung mit wohnfeldbasierter psychiatrischer Akutbehandlung ("Home Treatment"). Psychiatr Prax 38(03):123-128

Natarajan M, Srinivas J, Briscoe G, Forsyth S (2012) Community forensic psychiatry and the forensic mental health liaison model. Adv Psychiatr Treat 18(6):408-415
Nitschke J, Osterheider M, Mokros A (2011) Schizophreniforme Erkrankungen, Psychose und Tötungsdelikte: Die Bedeutung sozialtherapeutischer Maßnahmen zur Prävention von Delikten. Psychiatr Prax 38(02):82-86

Nitschke J, Sünkel Z, Mokros A (2018) Die Forensische Präventionsambulanz. Nervenarzt 89(9):1054-1062

Nitschke J, Sünkel Z, Mokros A (2020a) Die forensische Präventionsambulanz Ansbach. Nervenarzt 91(5):439-445

Nitschke J, Sünkel Z, Mokros A (2020b) Forensic prevention outpatient clinic: a treatment evaluation study. J Forensic Psychiatry Psychol 31(5):714-730

Piontek K, Kutscher SU, König A, Leygraf N (2013) Prädeliktische Behandlungswege schizophrener Patienten der forensischen Psychiatrie. Nervenarzt 84(1):55-64

Polhuis D, Lijten B, Mooij R (2017) Empowering community mental health teams by providing fo-rensic consultation. In: Proceedings of the 10th European Congress on. Violence in Clinical Psychiatry, Dublin, Ireland, S 339

Regierungsratsbeschluss (RRB) des Kantons Zürich Nr. 1048 (2011) https://www.zh.ch/de/politik-staat/gesetze-beschluesse/ beschluesse-des-regierungsrates/rrb/regierungsratsbeschluss1048-2011.html. Zugegriffen: 09.07.2021

Schröter R, Lambert M, Karow A, Lüdecke D, Gallinat J, Briken P (2021) Kurze Zündschnur: Ärger - Wut - Aggression. Forens Psychiatr Psychol Kriminol. https://doi.org/10.1007/s11757-02100655-y

Seliger M, Kröber HL (2008) Wurden schizophrene Maßregelpatienten zuvor in der Allgemeinpsychiatrie unzureichend behandelt? Forens Psychiatr Psychol Kriminol 2(2):46-53

Tribolet-Hardy F, Habermeyer E (2016) Schizophrenic patients between general and forensic psychiatry. Front Public Health. https://doi.org/10.3389/fpubh.2016.00135

Turner T, Salter M (2005) What is the role of a community forensic mental health team? Psychiatr Bull 29:352

Wolf A, Whiting D, Fazel S (2017) Violence prevention in psychiatry: an umbrella review of interventions in general and forensic psychiatry. J Forensic Psychiatry Psychol 28(5):659-673

Wössner G (2018) Fatales Spiel mit der Angst. Max-Planck-Forschung 2018:10-15

Zinkler M (2009) Allgemeine und forensische Psychiatrie - Wer kümmert sich um junge Menschen mit psychotischen Störungen? Psychiatr Prax 36:103-105 